\title{
Effects of increased wholegrain consumption on immune and inflammatory markers in healthy low habitual wholegrain consumers
}

Article

Accepted Version

Ampatzoglou, A., Williams, C. L., Atwal, K. K., Maidens, C. M., Ross, A. B., Thielecke, F., Jonnalagadda, S. S., Kennedy, O. B. and Yaqoob, P. (2016) Effects of increased wholegrain consumption on immune and inflammatory markers in healthy low habitual wholegrain consumers. European Journal of Nutrition, 55 (1). pp. 183-195. ISSN 1436-6215 doi: https://doi.org/10.1007/s00394-015-0836-y Available at https://centaur.reading.ac.uk/38990/

It is advisable to refer to the publisher's version if you intend to cite from the work. See Guidance on citing.

To link to this article DOI: http://dx.doi.org/10.1007/s00394-015-0836-y

Publisher: Springer

All outputs in CentAUR are protected by Intellectual Property Rights law, including copyright law. Copyright and IPR is retained by the creators or other copyright holders. Terms and conditions for use of this material are defined in the End User Agreement. 


\section{www.reading.ac.uk/centaur}

\section{CentAUR}

Central Archive at the University of Reading

Reading's research outputs online 


\section{Effects of increased wholegrain consumption on immune and inflammatory markers in}

healthy low habitual wholegrain consumers

Antonios Ampatzoglou ${ }^{1}$, Charlotte L. Williams ${ }^{1}$, Kiranjit K. Atwal ${ }^{1}$, Catherine M. Maidens ${ }^{1}$, Alastair B. Ross ${ }^{2,3}$, Frank Thielecke ${ }^{3,4}$, Satya S. Jonnalagadda ${ }^{5}$, Orla B. Kennedy ${ }^{1}$ and Parveen Yaqoob $^{1 *}$.

${ }^{1}$ Department of Food and Nutritional Sciences and Institute of Cardiovascular and Metabolic Research, University of Reading, Reading RG6 6AP, UK

2 Department of Chemical and Biochemical Engineering, Chalmers University of Technology, S-412 96 Gothenburg, Sweden

${ }^{3}$ Nestlé Research Centre, Nestec Ltd, Vers-chez-les-Blanc, 1000 Lausanne 26, Switzerland

${ }^{4}$ Cereal Partners Worldwide, Ch du Viaduc 1 - CH1000 Lausanne-Switzerland

${ }^{5}$ General Mills Bell Institute of Health and Nutrition, 9000 Plymouth Ave N, Golden Valley, MN 55427, USA

RUNNING TITLE: Wholegrain and immune function

KEY WORDS: Whole grain, immune function, inflammation, glucose metabolism WORD COUNT: 8,934; NUMBER OF FIGURES: 1; NUMBER OF TABLES: 5

* To whom correspondence should be addressed, e-mail: p.yaqoob@reading.ac.uk, phone: $+44(0) 1183788720$

Abbreviations used: 3DFD(s), 3-day food diary(-ies); AR(s), alkylresorcinol(s); CFDA-SE, carboxyfluorescein diacetate succinimidyl ester; CFSE, carboxyfluorescein succinimidyl ester; ConA, concanavalin A; CRP, C-reactive protein; DCD, diet compliance diary; FSC, forward scatter; GIP, gastric inhibitory polypeptide/glucose-dependent insulinotropic peptide; GLM, general linear model; GLP-1, glucagon-like peptide-1; MFI, mean fluorescence intensity; NK cells, natural killer cells; PAI-1, plasminogen activator inhibitor-1; PBMC(s), peripheral blood mononuclear cell(s); PI, propidium iodide; RG, refined grain; sIgA, secretory IgA; SSC, side scatter; Tcm, central memory $\mathrm{T}$ lymphocytes; Tem, effector memory $\mathrm{T}$ lymphocytes; Temra, $\mathrm{Ra}^{+}$effector memory $\mathrm{T}$ lymphocytes; $\mathrm{TNF}$, tumor necrosis factor; WBC(s), white blood cell(s); WG(s), wholegrain(s). 
1 ABSTRACT

2 Purpose

3 Wholegrain (WG) consumption is associated with reduced risk of cardiovascular disease, but

4 clinical data on inflammation and immune function is either conflicting or limited. The objective of

5 this study was to assess the impact of increasing WG consumption to at least $80 \mathrm{~g} / \mathrm{d}$ on markers of

6 inflammation and glucose metabolism and on phenotypic and functional aspects of the immune

7 system, in healthy, middle-aged adults with low habitual WG intake.

8 Methods

9 Subjects consumed a diet high in WG $(>80 \mathrm{~g} / \mathrm{d})$ or low in $\mathrm{WG}(<16 \mathrm{~g} / \mathrm{d}$, refined grain diet $)$ in a crossover study, with 6-week intervention periods, separated by a 4-week washout. Adherence to the dietary regimes was achieved by dietary advice and provision of a range of food products, with compliance verified through analysis of plasma alkylresorcinols (ARs).

\section{Results}

14 On the WG intervention, WG consumption reached $168 \mathrm{~g} / \mathrm{d}(P<0.001)$, accompanied by an 15 increase in plasma ARs $(P<0.001)$ and fibre intake $(P<0.001)$, without affecting other aspects of 16 dietary intake. On the WG arm there were trends for lower ex vivo activation of $\mathrm{CD}^{+}{ }^{+} \mathrm{T}$ cells and 17 circulating concentrations of IL-10, C-reactive protein, C-peptide, insulin and plasminogen 18 activator inhibitor- 1 . The percentage of $\mathrm{CD}^{+}$central memory $\mathrm{T}$ cells and circulating levels of adipsin tended to increase during the WG intervention.

\section{Conclusions}

21 Despite the dramatic increase in WG consumption, there were no effects on phenotypic or 22 functional immune parameters, markers of inflammation or metabolic markers.

Keywords

Alkylresorcinols, fibre, whole grain, immune, inflammation 


\section{INTRODUCTION}

Subclinical inflammation is associated with cardiovascular diseases (CVDs) [1], metabolic syndrome and type 2 diabetes [2,3]. Circulating concentrations of inflammatory markers, such as tumor necrosis factor (TNF)- $\alpha$, IL-6, IL-10 and C-reactive protein (CRP) are elevated in obese individuals and decrease with weight loss $[4,5]$ and may directly contribute to vascular injury, insulin resistance, and atherogenesis [6].

Wholegrain (WG) foods have been suggested to reduce the risk of CVD [7, 8], metabolic syndrome [8] and type 2 diabetes [7, 8]. However, the effects of WG consumption on health are still poorly understood. Some epidemiological studies have demonstrated an inverse association between WG consumption and CRP in non-diabetic [7,9] and diabetic [10] individuals, and between WG intake and elevated fasting levels of glucose [7], insulin [7, 11, 12], C-peptide [11] and leptin [11], but not all studies are consistent [11]. Intervention studies also present mixed data regarding effects of WG on CRP and other inflammatory markers. Some WG interventions (4-12 weeks) reported reductions in IL-6 [13] and CRP [14], especially in overweight and diabetic individuals or subjects with metabolic syndrome, while others report no effects of WG consumption ranging between 48 and 120 g/d on CRP, IL-6, PAI-1 [15-17], insulin [15, 16, 18, 19] or glucagon [18]. Interestingly, WG intakes were similar or higher $[15,16]$ in the studies that reported no effects, compared to those reporting positive effects, but it is notable that studies conducted in healthy volunteers tend to report no effect [15-18], whereas those conducted in non-healthy subjects tend to report positive effects $[14,20,21]$. This may be at least partly due to interventions in healthy subjects achieving a relatively modest level of WG intake (56-120 g/d in previous studies compared with an average intake of $168 \mathrm{~g} / \mathrm{d}$ in the current study). Some of these studies added WG to the diet rather than replacing refined grain products with WG products, or simply provided advice, whereas the current study specifically replaced RG products with WG products to achieve a high intake of WG compared with virtually no intake of WG during the RG period.

Currently, limited data is available on the impact of increased WG consumption on immune function. However, WGs are important sources of phytic acid [22], which may enhance the activity of natural killer (NK) cells [23, 24]. WGs also contain fructans, which have a degree of polymerisation between that of fructooligosaccharides' and inulin [25] and may modulate immune function [26, 27]. The objective of this randomized 6-week cross-over study was therefore to assess the effect of increasing WG consumption to at least $80 \mathrm{~g} / \mathrm{d}$ on markers of systemic inflammation and glucose metabolism and on phenotypic and functional aspects of the immune system in healthy, middle-aged adults, who had a habitual diet low in WG. The study was conducted in healthy individuals with a wide range of BMI in an effort to capture a population of likely consumers who 
span a wide range of body composition, but avoiding complications associated with diagnosed disease. While previous studies have focused on effects of WG on blood lipids, the current study examined immune and inflammatory markers, for which the issue of healthy vs non-healthy subjects has not yet been considered with respect to WGs.

\section{METHODS}

Subjects. Thirty-three healthy subjects (twelve male, twenty-one female), 40-65 years of age, with habitual WG consumption of less than $1 \frac{1 / 2}{2}$ serving or $24 \mathrm{~g} / \mathrm{d}$ and BMI $20-35 \mathrm{~kg} / \mathrm{m}^{2}$ were recruited into the study. Exclusion criteria included diagnosed diabetes, heart disease, stroke, vascular disease, inflammatory disease, renal, bowel, liver or pancreatic disease, medication for hyperlipidaemia, hypertension, hypercoagulation, inflammatory conditions or depression, prescribed aspirin, asthma, allergies, smoking, excessive consumption of alcohol (> 21 units/week for men, > 15 units/week for women), planned influenza vaccination or vaccination during the previous 12 months, consumption of prebiotics or probiotics or antibiotic treatment during the previous 3 months. Baseline characteristics of the subjects are shown in Table 1. This study was conducted according to the guidelines laid down in the Declaration of Helsinki and all procedures involving human subjects were approved by the University of Reading Research and Ethics Committee. Written informed consent was obtained from all subjects. The trial was registered (ISRCTN36521837).

Recruitment. Potential subjects responding to advertisements were sent a participant information sheet and those who were interested in participating completed a medical and lifestyle questionnaire either online or by telephone. Subjects consuming less than $24 \mathrm{~g} / \mathrm{d}$ of WG were identified using a FFQ, which was modified from Ross et al. [28] for use in the UK (48 items, 8 frequencies, with specified portion sizes for breakfast cereals, pasta and rice). Suitable subjects were then invited to the Hugh Sinclair Unit of Human Nutrition (Department of Food and Nutritional Sciences, University of Reading, UK) for a screening visit, where height, weight, waist circumference, and blood pressure were measured and a blood sample for a full blood count was taken.

Study design. During a 2-week run-in period, dietary assessment was conducted using 3-day food diaries (3DFDs) for any 3 consecutive days, which were also used to provide specific, tailored advice to achieve the WG target intakes. In consultation with a dietitian, subjects were advised to consume a diet high in WG $(>80 \mathrm{~g} / \mathrm{d})$ or low in WG $(<16 \mathrm{~g} / \mathrm{d}, \mathrm{RG}$ diet $)$ in a crossover study, with intervention periods lasting 6 week each, separated by a 4-week washout (Fig. 1). Subjects were 
randomized based on age, gender and BMI by a research assistant who was not involved in the analysis using covariate adaptive randomization software. Commercially available pasta, rice, snacks and breakfast cereals in both the WG and RG categories [29] were provided to volunteers every fortnight and specific advice given about substitution of habitually consumed products. Bread from a pre-agreed list was purchased by subjects and costs reimbursed upon production of a receipt. The values calculated for WG intake refer to the $\mathrm{g}$ amount of WG consumed (ie $20 \mathrm{~g}$ if $50 \mathrm{~g}$ of Uncle Ben's WG rice was consumed). The intakes were calculated by a dietician on an individual basis for every food included in the dietary records, using any available information relating to WG content of foods, or, in some cases, by contacting manufacturers. They were also calculated (much more simply) by the diet compliance diaries, which recorded consumption of the foods that were provided as part of the study. Data calculated using both methods allow comparison. This emphasizes the fact that during the WG intervention, almost all of the WG intake came from the products provided to the subjects, with very little being contributed by other products. This is perhaps not surprising, since the subjects were pre-selected to be very low habitual consumers of WG. While on the study, subjects were asked not to consume products containing prebiotics or probiotics. During each of the two intervention periods, subjects completed a 3DFD. All 3DFD were analyzed using Dietplan6 (Forestfield Software Ltd.).

Fasting blood and saliva samples were collected at the beginning and end of each of the intervention periods. Available data on which to perform power calculations was limited, so the sample size was based on data derived from interventions investigating the effect of prebiotics on phenotypic and functional immune parameters and the effect of prebiotics and WGs on the gut microbiota. A sample size of 33 subjects per group was sufficient to detect an $8 \%$ difference in NK cell activity, with a two-tailed significance level of $5 \%$ and a power of $80 \%$.

Saliva, plasma and serum samples. Saliva was collected by spitting into eppendorf tubes, centrifuged at $13,000 \mathrm{x} \mathrm{g}$ for $10 \mathrm{~min}$ at $4^{\circ} \mathrm{C}$, and the supernatants stored at $-20^{\circ} \mathrm{C}$ for $<15$ months before further analysis. Plasma was prepared from blood collected in EDTA tubes by centrifugation at $2000 \mathrm{x} \mathrm{g}$ for $15 \mathrm{~min}$ at room temperature (RT) and serum was prepared from blood collected in serum sep clot activator tubes by centrifugation at $1800 \mathrm{x} g$ for $10 \mathrm{~min}$ at RT. Samples were frozen at $-20^{\circ} \mathrm{C}$ within $1 \mathrm{~h}$ from collection until further analysis.

\section{Preparation of peripheral blood mononuclear cells (PBMCs). Heparinised whole blood (15 ml)} was layered onto Lympholyte-H (25 ml; Cedarlane laboratories, Texas, USA), before centrifuging the samples at $550 \mathrm{x} \mathrm{g}$ for $15 \mathrm{~min}$ at RT and collecting the PBMC-rich interface. PBMCs were washed in PBS (Oxoid, Hampshire, UK), resuspended in $2.5 \mathrm{ml}$ of RPMI 1640 and layered onto 2.5 
$\mathrm{ml}$ Lympholyte-H. Samples were then centrifuged once more at $550 \mathrm{x} \mathrm{g}$ for $15 \mathrm{~min}$ at RT, before collecting the PBMC-rich interface and washing with PBS. PBMCs were resuspended in complete medium (RPMI 1640 with HEPES and L-Glutamine medium containing $100 \mathrm{U} / \mathrm{ml}$ penicillin and $0.1 \mathrm{mg} / \mathrm{ml}$ streptomycin), counted in a $\mathrm{Z1}^{\mathrm{TM}}$ series Coulter Counter ${ }^{\circledR}$ (Beckman Coulter, High Wycombe, UK) and adjusted to $5 \times 10^{6}$ cells $/ \mathrm{ml}$.

Analysis of plasma alkylresorcinols (ARs). To assess compliance, plasma ARs were analyzed by extraction with diethyl ether and normal phase liquid chromatography/tandem mass spectrometry (LC-MS/MS) after extraction with diethyl ester [30] modified to a $100 \mathrm{~mm}$ x $2 \mathrm{~mm}$ column and a run time of $8 \mathrm{~min}$. Matrix matched calibration was used, and two control samples were run in triplicate with each batch. Control samples and calibration curve standards were run in random order throughout each sequence. Intra-batch repeatability for each control plasma sample was $<10$ $\%$ and inter-batch repeatability $<15 \%$.

White Blood Cell (WBC) counts. Whole blood was diluted 1:501 with ISOTON II (Beckman Coulter, High Wycombe, UK), erythrocytes were lysed using ZAP-OGLOBIN II. (Beckman Coulter) and samples were counted on a $\mathrm{Z1} 1^{\mathrm{TM}}$ series Coulter Counter®. The average of three measurements was recorded.

Enumeration of lymphocyte subsets using TruCount ${ }^{\mathrm{TM}}$ tubes. Absolute counts of $\mathrm{T}$ lymphocytes, B lymphocytes and NK cells were performed by flow cytometry using the BD Multitest $^{\mathrm{TM}}$ CD3 FITC/CD16 ${ }^{+} \mathrm{CD} 56$ PE/CD45 PerCP/CD19 APC with BD Trucount ${ }^{\mathrm{TM}}$ Tubes (BD Biosciences, Oxford, UK). Samples were analysed on a BD FACSCanto II flow cytometer (BD Biosciences).

Naïve and memory T lymphocyte subsets. Whole blood in EDTA tubes was stained with a mixture of APC-Cy7 mouse anti-human CD3, FITC mouse anti-human CD4, PerCP-Cy5.5 mouse anti-human CD8, PE-Cy7 mouse anti-human CD45RA and PE rat anti-human CD197 (CCR7) (BD Biosciences, Oxford, UK) in the dark for $45 \mathrm{~min}$ at RT. Erythrocytes were lysed using BD Pharm Lyse $^{\mathrm{TM}}$ lysing buffer (BD Biosciences, Oxford, UK), incubating in the dark for 20 min at RT, before washing twice with BD CellWASHTM (BD Biosciences). Samples were analysed on a BD FACSCanto II flow cytometer. Results are reported as percentages of parent $\mathrm{CD}^{+}$and $\mathrm{CD} 4^{+} \mathrm{T}$ lymphocyte subsets. 
Ex vivo phagocytosis monocytes and granulocytes. Monocyte and granulocyte phagocytosis was assessed using the PHAGOTEST® kit (Glycotype Biotechnology, Heidelberg, Germany); both the percentage of monocytes and granulocytes engaged in phagocytosis of opsonised FITC-labelled Escherichia coli bacteria and mean fluorescence intensity (MFI), reflecting degree of phagocytosis, were recorded. Samples were analysed on a BD FACSCanto II flow cytometer within 60 min.

Ex vivo T cell activation. Heparinised whole blood was diluted 1:10 with complete medium, stimulated with concanavalin A (Sigma-Aldrich, Poole, UK) or CytoStim (Miltenyi Biotec, Surrey, $\mathrm{UK}$ ) at final concentrations of $25 \mu \mathrm{g} / \mathrm{ml}$ and $0.1 \mu \mathrm{l} / \mathrm{ml}$ respectively and incubated at $37^{\circ} \mathrm{C}, 5 \% \mathrm{CO}_{2}$ for $4 \mathrm{~h}$. Samples were washed once with EDTA buffer (2 mM EDTA in PBS, containing 0.5\% v/v BSA stain buffer) before staining with a mixture of APC-Cy7 mouse anti-human CD3, FITC mouse anti-human CD4, PE mouse anti-human CD8 and APC mouse anti-human CD69 (BD Biosciences, Oxford, UK) in the dark for 45 min at RT. Erythrocytes were lysed using BD Pharm Lyse ${ }^{\mathrm{TM}}$ lysing buffer, incubating in the dark for $20 \mathrm{~min}$ at RT, before washing twice with BD CellWASH ${ }^{\mathrm{TM}}$. Finally, 7-AAD (BD Biosciences, Oxford, UK) was added as a viability stain and samples were analysed on a BD FACSCanto II flow cytometer.

NK cell activity assay. K562 cells were stained with $0.2 \%$ Trypan Blue (Sigma-Aldrich, Poole, UK) and counted using a haemocytometer before removing $5 \times 10^{6}$ live K562 cells from the suspension. Cells were washed twice in PBS and stained with carboxyfluorescein diacetate succinimidyl ester (CFDA-SE, $100 \mu \mathrm{g} / \mathrm{ml}$, Sigma-Aldrich) in dimethyl sulfoxide (Sigma-Aldrich) at $37^{\circ} \mathrm{C}, 5 \% \mathrm{CO}_{2}$ for $45 \mathrm{~min}$, during which CFDA-SE was intracellularly converted to carboxyfluorescein succinimidyl ester (CFSE). CFSE-labelled K562 cells were washed twice in PBS, re-suspended and diluted 1:100 in complete medium. PBMCs were then incubated together with the K562 cells at ratios of 100:1, 50:1, 25:1, 12.5:1 and 0:1 (PBMC:K562) at $37^{\circ} \mathrm{C}, 5 \% \mathrm{CO}_{2}$ for $2 \mathrm{~h}$. Propidium iodide ( $1 \mathrm{mg} / \mathrm{ml}$, Sigma-Aldrich) in PBS was used to stain dead K562 cells, prior to flow-cytometric analysis in a FACSCanto II (BD Biosciences).

Serum cytokine analysis. Serum concentrations of IL-1 $\beta$, IL-6, IL-8, IL-10 and TNF- $\alpha$ were analysed by Luminex ${ }^{\circledR} 100^{\mathrm{TM}}$ (Luminex Corporation), using a high sensitivity multiplex cytokine Kit (R\&D Systems, Abingdon, UK), according to the manufacturer's instructions. Standard curves were generated by the Luminex ${ }^{\circledR}$ software (mean $R^{2}=0.9991$ ). All samples for individual subjects were analysed within a single plate and the intra-assay CV for the Luminex platform was 5.9\%. 
CRP analysis. CRP concentrations were analysed by an Automatic Analyzer ILab 600 using enzyme based kits and appropriate quality controls (Instrumentation Laboratory Ltd.). All samples for individual subjects were analysed within a single batch and the intra-assay $\mathrm{CV}$ was $1.6 \%$.

Salivary secretory IgA (sIgA). Salivary sIgA was analysed using Immunodiagnostic sIgA ELISA kit (Oxford Biosystems, Oxford, UK), in accordance with the manufacturer's instructions, All samples for individual subjects were analysed within a single plate and the intra-assay CV was $9.0 \%$.

Metabolic and inflammatory markers. Serum concentrations of C-Peptide, ghrelin, gastric inhibitory polypeptide (GIP), glucagon-like peptide-1 (GLP-1), glucagon, insulin, leptin, PAI-1, resistin, visfatin, adiponectin and adipsin were analysed by Luminex ${ }^{\circledR} 100^{\mathrm{TM}}$, using the Bio-Plex Pro Human Diabetes 10-Plex Assay and Bio-Plex Pro Human Diabetes Adipsin and Adiponectin Assays (Bio-Rad Laboratories) according to the manufacturer's instructions. All samples for individual subjects were analysed within a single plate and the intra-assay CV was $16.6 \%$.

Statistical analysis. Analysis of the data acquired by flow cytometry was performed by BD FACSDiva Software (BD Biosciences) and statistical analysis was carried out on Minitab, version 15 (Minitab Ltd. State College, PA). Data are means and SD or SEM at baseline and end of intervention (within each arm), as well as difference from the within-arm baseline ( $\Delta$ intervention) for both interventions. Data were normally distributed, as confirmed by the Kolmogorov-Smirnov normality test. To assess whether the order of the intervention arms had an effect on the data, baseline means were compared using two sample T-tests and no statistical differences were found. With the exceptions mentioned below, a general linear model (GLM) was used to analyze the data, in which intervention delta and gender were specified as fixed model parameters and starting diet as a random parameter, while age and baseline measurements of BMI, systolic and diastolic blood pressure, body fat percentage, waist circumference, alcohol consumption, plasma ARs, habitual fibre and WG consumption, CRP and blood biochemistry (i.e. total cholesterol, triglycerides, LDL and HDL cholesterol and glucose) were listed as covariates. The analysis of 3DFD and ARs was performed using a single factor GLM and further comparisons made using Tukey simultaneous tests. Spearman's $\rho$ correlation coefficient between studied parameters and ARs, fibre intake and WG intake were calculated and $P$-values reported. In all cases, the level of significance was set at $5 \%$ and $10 \%$ for effects and trends, respectively.

\section{RESULTS}




\section{Dietary analysis, assessment of WG intake and plasma ARs}

A habitual diet low in WG intake was a prerequisite for subjects taking part in this study and two methods were used to assess it; the FFQ at screening and the 3DFD during the run-in period. Subjects were recruited on the basis of the FFQ, although comparison with the 3DFD suggests that the FFQ slightly but significantly underestimated habitual consumption of WG $(P<0.001)$ (data not shown). Using data from the 3DFD administered during the run-in period, recruited subjects consumed on average $28 \mathrm{~g} / \mathrm{d}$ of WG, which was slightly higher than the threshold of $24 \mathrm{~g} / \mathrm{d}$. Nevertheless, the substitution of habitual grain-based foods with the provided WG varieties during the WG intervention period resulted in consumption of WG far exceeding the target of $80 \mathrm{~g} / \mathrm{d}$ and reaching an average of $168 \mathrm{~g} / \mathrm{d}$, with a range of 67-335 g/d (Table 2). In contrast, WG consumption during the RG period was $0.1 \mathrm{~g} / \mathrm{d}$ on average (Table 2). During the WG intervention, wheat was the major contributor to the increased WG intake, providing $59 \%$ of the WG consumed, while oats, rice and corn contributed $40 \%$ in total. Increased WG consumption was accompanied by a statistically significant increase in plasma ARs and total fibre intake, but there was no effect on other aspects of dietary intake (Table 2).

Effect of WG intervention on absolute numbers of WBCs, B and T lymphocytes and NK cells and on naïve and memory $T$ lymphocyte subsets

There was no effect of the WG intervention on absolute counts of WBCs, B and T lymphocytes or NK cells (data not shown) or on naïve or memory cell subpopulations of $\mathrm{CD}^{+}$and $\mathrm{CD}^{+} \mathrm{T}$ lymphocytes (Table 3). However, there was a trend towards an increase in CD4 ${ }^{+} \mathrm{Tcm}$ cells during the WG intervention $(P=0.06)$. Modest positive correlations were observed between plasma ARs, but not WG intake (Spearman's $\rho, P)$, and absolute numbers of B $(0.19,0.03)$ and T $(0.18,0.05)$ lymphocytes and NK cells $(0.23,0.01)$.

Effect of WG intervention on ex vivo phagocytic ability of monocytes and granulocytes, $T$ cell activation and NK cell activity

The intervention had no effect on phagocytosis by monocytes or granulocytes (data not shown). There was also no effect on the expression of $\mathrm{CD} 69$ by $\mathrm{CD}^{+}$or $\mathrm{CD}^{+} \mathrm{T}$ lymphocyte subsets following ex vivo stimulation of whole blood cultures (Table 4), or on NK cell activity towards K562 cells (data not shown). ConA-stimulated expression of CD69 on CD4 ${ }^{+}$cells tended to be higher during the RG period than the WG period $(P=0.08)$.

\section{Effect of WG intervention on serum cytokines, CRP and salivary SIgA}


There was no effect of the intervention on serum concentrations of cytokines or CRP (Table 5) or on salivary $\operatorname{sIgA}$ (data not shown). There was a trend towards a reduction in IL-10 and CRP during the WG intervention ( $P=0.08$ and 0.10 , respectively) and a modest negative correlation between IL-1 $\beta$ and total fibre intake (Spearman's $\rho=-0.21, P=0.04$ ). Despite the inverse trend with WG intake, IL-10 was positively correlated with plasma ARs (Spearman's $\rho=0.19, P=0.04$ ).

\section{Effect of WG intervention on metabolic markers}

There was no effect of WG on any of the metabolic markers assessed (Table 6), although Cpeptide, insulin and PAI- 1 tended to be higher during the RG period than the WG period $(P=0.10$, 0.08 and 0.07 , respectively). In contrast, there was a trend towards a reduction in adipsin during the RG period compared with the WG period $(P=0.07)$. Adipsin was also positively correlated with plasma ARs (Spearman's $\rho=0.22, P=0.01$ ), but not with WG.

\section{DISCUSSION}

This intervention resulted in a substantial increase in WG consumption in habitual low consumers of WG to an average of $168 \mathrm{~g} / \mathrm{d}$ (a 500\% increase), which was associated with an increase in plasma ARs. Despite the increase in WG consumption, there were no effects on phenotypic or functional immune parameters, markers of inflammation or metabolic markers.

ARs are phenolic lipids that have been proposed as potential biomarkers of WG intake [31] and are present in the bran of rye, wheat and barley, but not in the edible parts of other cereals. In line with published data [32], total AR concentrations were below $60 \mathrm{nM}$ at baseline and during the RG arm and the mean concentration after 6 weeks of WG intervention was $161 \mathrm{nmol} / \mathrm{L}$. This is at the lower end of the previously reported range of $142-847 \mathrm{nmol} / \mathrm{L}$ following a WG intervention of a similar magnitude [32].

In the current study, there were no effects of WG consumption on absolute counts of WBC, B and T lymphocytes, NK cells, naïve and memory cell subsets of $\mathrm{CD}^{+}$and $\mathrm{CD}^{+} \mathrm{T}$ lymphocytes, phagocytic ability of monocytes and granulocytes, activation (expression of CD69) of $\mathrm{T}$ lymphocytes after ex vivo stimulation of whole blood, activity of NK cells or concentration of saliva sIgA. While there was a trend towards increased activation of $\mathrm{CD}^{+} \mathrm{T}$ cells after stimulation with ConA during the RG period relative to the WG period and a trend towards increased percentage of $\mathrm{CD}^{+} \mathrm{Tcm}$ cells during the WG intervention, the lack of consistent effects on immune function suggest that these are not meaningful and cannot be interpreted as either positive or negative effects. There is currently very limited data on the impact of increased WG consumption on immune function. Single measures of immune function are notoriously difficult to interpret with respect to biological or clinical relevance, but the use of a panel of markers has the potential to identify 
consistent effects, which is arguably a more appropriate approach to investigating the effects of dietary components on immune function [33]. The primary immune outcomes in the current study were immune phenotyping and functional analysis and the secondary outcomes were the circulating metabolic and inflammatory markers.

WGs, and rice, corn and wheat in particular, are important sources of phytic acid [22, 34], a metal chelator which may enhance the activity of NK cells $[23,24]$. WGs also contain arabinoxylans, $\beta$ glucans and fructans, which have structural similarities with inulin and fructooligosaccharides [25], both of which have been shown to have beneficial effects on parameters of immune function. For example, clinical interventions in elderly adults $(>65 y)$ demonstrate that inulin and fructooligosaccharides increase numbers of in peripheral blood B cells [26], T cells [34], and CD4 ${ }^{+}$ and $\mathrm{CD}^{+} \mathrm{T}$ cell subsets [35], decrease numbers of memory $\mathrm{CD}^{+} \mathrm{T}$ cells [26] and improve NK cell activity [27]. However, in similar studies in young healthy individuals [35] or cancer and polypectomised patients [36], there was little or no effect of inulin, as part of a synbiotic [36] or other prebiotics [35] on phagocytic activity of neutrophils [35, 36] and monocytes [36] or activity [36] and numbers of NK cells [35].

Increased WG consumption had no effect on either serum cytokines or on CRP levels, although there were trends towards decreased IL-10 and CRP concentrations during the WG compared to the RG period. Based on epidemiological data, WG consumption has been inversely associated with inflammatory markers, and particularly CRP [37]. However, intervention studies do not always demonstrate a clear effect. Martinez et al. [13] reported reductions in IL-6 and CRP concentrations (although the latter did not reach statistical significance) after 4 weeks of consumption of $60 \mathrm{~g} / \mathrm{d}$ of WG rice and barley. Katcher et al. [14] reported a $38 \%$ reduction in CRP following a WG diet for 12 weeks. In these studies, WG products were either added to the diet instead of replacing the existing RG products [13] or subjects were given dietary advice either to avoid WG or to obtain all of their grain servings from WG [14]. Importantly, these studies were either conducted in obese volunteers with metabolic syndrome [14] or reductions in inflammatory markers were more significant in overweight subjects [13], suggesting that health status of the subject might be a key factor determining the outcome. In another study, both a WG (60 g/d of WG) and a RG (4 g/d of WG) diet resulted in reductions between 22 and $42 \%$ in TNF- $\alpha$, IL-1 $\beta$, and IL- 6 after 6 weeks in adolescents [38], but some intervention studies reported no effects of WG on CRP or IL-6 after 2 [39] 6 [15], 8 [16], 12 [40] or 16 [16] weeks following a WG diet (between 48 and 150 g/d of WG). A number of aspects of health status may influence the impact of WG consumption on health outcomes, including presence of metabolic syndrome or type 2 diabetes, initial concentration of the studied inflammatory markers, intervention duration, cereal type and perhaps even particular cultivar and processing. Variations in these factors may well underlie discrepancies between and 
within epidemiological and intervention studies. In addition, glycemic index (GI) of the foods selected for the intervention may be an important factor for consideration. A number of the WG foods provided had a medium or high GI value [41], and GI has been demonstrated to modulate inflammatory markers [42], perhaps even more so than WG. The desire to provide a wide range of acceptable foods to achieve a high total WG intake therefore conflicts with the potential necessity to limit the WG foods to those which are low GI, and the overall impact of WG vs GI on inflammation is not yet clear.

WG consumption had little effect on the metabolic markers examined in the current study, although there was a tendency for C-peptide, insulin and PAI-1 to decrease, while adipsin tended to increase during the WG period relative to the RG period. Reviewing observational studies and clinical trials, Koh-Banerjee and Rimm [43] concluded that WG intake is inversely associated with insulin, Cpeptide and leptin concentrations. Moreover, based on epidemiological studies, PAI-1 has been related to RG and inversely associated with WG [9]. Generally, however, WG intervention studies have had mixed results in terms of markers related to glucose metabolism. Following WG interventions of 6 or 12 weeks, insulin, leptin and PAI-1 were significantly decreased in overweight or obese hyperinsulinaemic adults [20], patients with early-stage prostate cancer [21] and obese adults with metabolic syndrome [14], respectively. In these studies WG was either based solely on rye [21] or consumed in a variety of products $[14,20]$, similar to the current study. However, in the study of Katcher et al. [14], total energy was restricted and the reported reduction in PAI-1 was attributed to weight loss, rather than increased consumption of WG. Indeed, loss of body weight is directly associated with lowering PAI-1 and also leptin and resistin concentrations [5]. Another study, reported a decline in adiponectin levels in diabetic patients, after a high-carbohydrate, lowfibre, RG wheat meal in comparison to a high-carbohydrate, high-fibre WG wheat meal [44]. On the other hand, a number of studies in healthy individuals report no effects of WG on PAI-1 [15, 16, 25], insulin [15, 16, 18, 19] or glucagon [18] after 4 [17], 6 [15, 18], 8 [16], 12 [19] or 16 [16] weeks of consuming whole meal rye bread [17], WG wheat sourdough bread [18], high-fibre oat or wheat cereal [19] or a variety of WG products [15, 16], where WG intake ranged from 56 to $120 \mathrm{~g} / \mathrm{d}$ $[15,16]$. Notably, most studies show no effects of WG on metabolic markers in healthy subjects (n $=28-316)$ [15-19], while clinical interventions tend to report significant effects $(\mathrm{n}=11-60)[14,20$, $21,44]$. In the current study, there were no effects of the WG intervention on body weight, BMI, body fat percentage or waist circumference [29] and there was no influence of body composition on immunological parameters.

Power is an important consideration in evaluation of the evidence for health effects of WGs; inconsistency in previous data and uncertainty with respect to effect size meant that it was difficult to conduct a well-justified power calculation and the sample size in the current study was based on 
interventions investigating the effect of prebiotics on phenotypic and functional immune parameters and the effect of prebiotics and WGs on the gut microbiota. The numbers of participants in previously published studies have ranged from 17 to and 316, with the majority including 50 subjects or fewer. Thus, although the current study demonstrated no effects in healthy subjects, the potential for WGs to improve inflammatory markers and markers of glucose metabolism needs to be further examined, perhaps in subjects pre-selected for inflammatory status, and almost certainly with larger sample sizes.

Processing and the physical properties, structure and composition of the cereal grain, especially with regard to amount and type of fibre, may be important considerations in WG intervention studies. Anson et al. [45] demonstrated that bio-processing of bran in WG wheat bread resulted in increased bio-availability of phenolic acids and consumption of the processed wheat bread decreased the ratio of pro-inflammatory to anti-inflammatory cytokines in endotoxin-stimulated blood ex vivo compared with non-processed bread. However, evidence for effects of processing is generally limited. The role of the amount and type of fibre in WG foods, is better understood, particularly with respect to carbohydrate metabolism [46]. It is well known, for example, that soluble fibre improves control of blood glucose and insulin levels [39]. Wheat contains relatively less soluble and more insoluble fibre than oats, rye or barley, which may partially explain differences in the outcomes of studies where different sources of WG were used. In the current study, $59 \%$ of the WG intake was derived from wheat, and it was this source of WG which increased plasma ARs. It was notable that changes in some biomarkers correlated well with plasma AR concentrations, but not WG intake (eg adipsin), and it is tempting to speculate that this might be related to effects of wheat vs oats, rye or barley. Plasma AR concentrations may also reflect the extent of absorption of phytochemicals from WG, and better represent actual exposure of the body to WG components, than estimates of WG intake.

In conclusion, despite the dramatic increase in WG consumption in habitual low consumers of WG, there were no effects on phenotypic or functional immune parameters, markers of inflammation or metabolic markers. Health status of subjects, statistical power, physical and structural properties of the WGs and processing conditions may be important factors to consider in future studies.

\section{Acknowledgements}

AA, FT, SSJ, OBK and PY designed the research. AA, KKA and CMM recruited the volunteers and conducted the clinical visits. KKA and CMM analyzed the 3DFDs. AA and CLW processed clinical samples and analyzed biochemical parameters and ABR analyzed plasma ARs. AA performed the statistical analysis. AA, PY, ABR and FT wrote the paper and PY had primary 
407 responsibility for the final manuscript. All authors read and approved the manuscript. We thank 408 Mrs. Jan Luff for assisting with recruitment.

409

410 Conflict of interest

411 This work was funded by Cereal Partners Worldwide. AA, CLM, KKA, CMM, OBK and PY have 412 no conflicts of interest. ABR was an employee of Nestlé at the time of this research and FT and SSJ 413 are employees of Cereal Partners Worldwide and General Mills, respectively. Nestlé, Cereal 414 Partners Worldwide and General Mills all produce a range of whole grain food products. 
417 1. Libby P, Ridker PM (2004) Inflammation and atherosclerosis: Role of C-reactive protein in risk assessment. Am J Med 116:9-16. doi Doi 10.1016/J.Amjmed.2004.02.006.

2. Hu FB, Meigs JB, Li TY, Rifai N, Manson JE (2004) Inflammatory markers and risk of developing type

3. Pradhan AD, Manson JE, Rifai N, Buring JE, Ridker PM (2001) C-reactive protein, interleukin 6, and risk of developing type 2 diabetes mellitus. Jama-J Am Med Assoc 286:327-334. doi Doi 10.1001/Jama.286.3.327.

4. Derosa G, Fogari E, D'Angelo A, Bianchi L, Bonaventura A, Romano D, Maffioli P (2013) Adipocytokine Levels in Obese and Non-obese Subjects: an Observational Study. Inflammation 36:914-920. doi Doi 10.1007/S10753-013-9620-4.

5. Calder PC, Ahluwalia N, Brouns F, Buetler T, Clement K, Cunningham K, Esposito K, Jonsson LS, Kolb H, Lansink M, Marcos A, Margioris A, Matusheski N, Nordmann H, O'Brien J, Pugliese G, Rizkalla S, Schalkwijk C, Tuomilehto J, Warnberg J, Watzl B, Winklhofer-Roob BM (2011) Dietary factors and low-grade inflammation in relation to overweight and obesity. British Journal of Nutrition 106:S1-+. doi Doi 10.1017/S0007114511005460.

6. Lau DCW, Dhillon B, Yan HY, Szmitko PE, Verma S (2005) Adipokines: molecular links between obesity and atheroslcerosis. Am J Physiol-Heart C 288:H2031-H2041. doi Doi 10.1152/Ajpheart.01058.2005.

7. Lutsey PL, Jacobs DR, Kori S, Mayer-Davis E, Shea S, Steffen LM, Szklo M, Tracy R (2007) Whole grain intake and its cross-sectional association with obesity, insulin resistance, inflammation, diabetes and subclinical CVD: The MESA study. British Journal of Nutrition 98:397-405. doi Doi $10.1017 /$ S0007114507700715.

8. McKeown NM, Meigs JB, Liu SM, Wilson PWF, Jacques PF (2002) Whole-grain intake is favorably associated with metabolic risk factors for type 2 diabetes and cardiovascular disease in the Framingham Offspring Study. American Journal of Clinical Nutrition 76:390-398.

9. Masters RC, Liese AD, Haffner SM, Wagenknecht LE, Hanley AJ (2010) Whole and Refined Grain Intakes Are Related to Inflammatory Protein Concentrations in Human Plasma. Journal of Nutrition 140:587-594. doi Doi 10.3945/Jn.109.116640.

10. Qi L, Van Dam RM, Liu SM, Franz M, Mantzoros C, Hu FB (2006) Wholeo-grain, bran, and cereal fiber intakes and markers of systemic inflammation in diabetic women. Diabetes Care 29:207-211. doi Doi 10.2337/Diacare.29.02.06.Dc05-1903.

11. Jensen MK, Koh-Banerjee P, Franz M, Sampson L, Gronbaek M, Rimm EB (2006) Whole grains, bran, and germ in relation to homocysteine and markers of glycemic control, lipids, and inflammation. American Journal of Clinical Nutrition 83:275-283.

12. Liese AD, Roach AK, Sparks KC, Marquart L, D'Agostino RB, Mayer-Davis EJ (2003) Whole-grain intake and insulin sensitivity: the Insulin Resistance Atherosclerosis Study. American Journal of Clinical Nutrition 78:965-971.

13. Martinez I, Lattimer JM, Hubach KL, Case JA, Yang JY, Weber CG, Louk JA, Rose DJ, Kyureghian G, Peterson DA, Haub MD, Walter J (2013) Gut microbiome composition is linked to whole graininduced immunological improvements. Isme J 7:269-280. doi Doi 10.1038/Ismej.2012.104.

14. Katcher HI, Legro RS, Kunselman AR, Gillies PJ, Demers LM, Bagshaw DM, Kris-Etherton PM (2008) The effects of a whole grain-enriched hypocaloric diet on cardiovascular disease risk factors in men and women with metabolic syndrome. American Journal of Clinical Nutrition 87:79-90.

15. Andersson A, Tengblad S, Karlstrom B, Kamal-Eldin A, Landberg R, Basu S, Aman P, Vessby B (2007) Whole-grain foods do not affect insulin sensitivity or markers of lipid peroxidation and inflammation in healthy, moderately overweight subjects. Journal of Nutrition 137:1401-1407.

16. Brownlee IA, Moore C, Chatfield M, Richardson DP, Ashby P, Kuznesof SA, Jebb SA, Seal CJ (2010) Markers of cardiovascular risk are not changed by increased whole-grain intake: the WHOLEheart study, a randomised, controlled dietary intervention. British Journal of Nutrition 104:125-134. doi Doi 10.1017/S0007114510000644. 
17. Turpeinen AM, Juntunen K, Mutanen M, Mykkanen H (2000) Similar responses in hemostatic factors after consumption of wholemeal rye bread and low-fiber wheat bread. European Journal of Clinical Nutrition 54:418-423. doi Doi 10.1038/Sj.Ejcn.1600975.

18. Tucker AJ, MacKay KA, Robinson LE, Graham TE, Bakovic M, Duncan AM (2010) The effect of whole grain wheat sourdough bread consumption on serum lipids in healthy normoglycemic/normoinsulinemic and hyperglycemic/hyperinsulinemic adults depends on presence of the APOE E3/E3 genotype: a randomized controlled trial. Nutr Metab 7. doi Artn 37

Doi 10.1186/1743-7075-7-37.

19. Davy BM, Davy KP, Ho RC, Beske SD, Davrath LR, Melby CL (2002) High-fiber oat cereal compared with wheat cereal consumption favorably alters LDL-cholesterol subclass and particle numbers in middle-aged and older men. American Journal of Clinical Nutrition 76:351-358.

20. Pereira MA, Jacobs DR, Pins JJ, Raatz SK, Gross MD, Slavin JL, Seaquist ER (2002) Effect of whole grains on insulin sensitivity in overweight hyperinsulinemic adults. American Journal of Clinical Nutrition 75:848-855.

21. Moazzami AA, Zhang JX, Kamal-Eldin A, Aman P, Hallmans G, Johansson JE, Andersson SO (2011) Nuclear Magnetic Resonance-Based Metabolomics Enable Detection of the Effects of a Whole Grain Rye and Rye Bran Diet on the Metabolic Profile of Plasma in Prostate Cancer Patients. Journal of Nutrition 141:2126-2132. doi Doi 10.3945/Jn.111.148239.

22. Fardet A (2010) New hypotheses for the health-protective mechanisms of whole-grain cereals: what is beyond fibre? Nutrition Research Reviews 23:65-134. doi Doi $10.1017 /$ S0954422410000041.

23. Reddy BS (1999) Prevention of colon carcinogenesis by components of dietary fiber. Anticancer Res 19:3681-3683.

24. Baten A, Ullah A, Tomazic VJ, Shamsuddin AM (1989) Inositol-Phosphate-Induced Enhancement of Natural-Killer Cell-Activity Correlates with Tumor Suppression. Carcinogenesis 10:1595-1598. doi Doi 10.1093/Carcin/10.9.1595.

25. Haska L, Nyman M, Andersson R (2008) Distribution and characterisation of fructan in wheat milling fractions. J Cereal Sci 48:768-774. doi Doi 10.1016/J.Jcs.2008.05.002.

26. Langkamp-Henken B, Wood SM, Herlinger-Garcia KA, Thomas DJ, Stechmiller JK, Bender BS, Gardner EM, DeMichele SJ, Schaller JP, Murasko DM (2006) Nutritional formula improved immune profiles of seniors living in nursing homes. J Am Geriatr Soc 54:1861-1870. doi Doi 10.1111/J.15325415.2006.00982.X.

27. Bunout D, Barrera G, Hirsch S, Gattas V, de la Maza MP, Haschke F, Steenhout $P$, Klassen $P$, Hager $C$, Avendano M, Petermann M, Munoz C (2004) Effects of a nutritional supplement on the immune response and cytokine production in free-living Chilean elderly. JPEN. Journal of parenteral and enteral nutrition 28:348-354.

28. Ross AB, Pineau N, Kochhar S, Bourgeois A, Beaumont M, Decarli B (2009) Validation of a FFQ for estimating whole-grain cereal food intake. British Journal of Nutrition 102:1547-1551. doi Doi 10.1017/S0007114509990845.

29. Ampatzoglou A AK, Maidens CM, Williams CL, Ross AB, Thielecke F, Jonnalagadda SS, Kennedy OB, Yaqoob P. (2015) Increased whole grain consumption does not affect blood biochemistry, body composition or gut microbiology in healthy low habitual whole grain consumers. Journal of Nutrition:in press.

30. Ross AB, Redeuil K, Vigo M, Rezzi S, Nagy K (2010) Quantification of alkylresorcinols in human plasma by liquid chromatography/tandem mass spectrometry. Rapid Commun Mass Sp 24:554-560. doi Doi 10.1002/Rcm.4409.

31. Ross AB, Kamal-Eldin A, Aman P (2004) Dietary alkylresorcinols: Absorption, bioactivities, and possible use as biomarkers of whole-grain wheat- and rye-rich foods. Nutr Rev 62:81-95. doi Doi 10.1301/Nr.2004.Mar.81-95.

32. Ross AB, Bruce SJ, Blondel-Lubrano A, Oguey-Araymon S, Beaumont M, Bourgeois A, NielsenMoennoz C, Vigo M, Fay LB, Kochhar S, Bibiloni R, Pittet AC, Emady-Azar S, Grathwohl D, Rezzi S (2011) A whole-grain cereal-rich diet increases plasma betaine, and tends to decrease total and 
33. Albers R, Bourdet-Sicard R, Braun D, Calder PC, Herz U, Lambert C, Lenoir-Wijnkoop I, Meheust A, Ouwehand A, Phothirath P, Sako T, Salminen S, Siemensma A, van Loveren H, Sack U (2013) Monitoring immune modulation by nutrition in the general population: identifying and substantiating effects on human health. British Journal of Nutrition 110:S1-S30. doi Doi $10.1017 /$ S0007114513001505.

34. Guigoz Y, Rochat F, Perruisseau-Carrier G, Rochat I, Schiffrin EJ (2002) Effects of oligosaccharide on the faecal flora and non-specific immune system in elderly people. Nutrition Research 22:13-25. doi Doi 10.1016/S0271-5317(01)00354-2.

35. Seidel C, Boehm V, Vogelsang H, Wagner A, Persin C, Glei M, Pool-Zobel BL, Jahreis G (2007) Influence of prebiotics and antioxidants in bread on the immune system, antioxidative status and antioxidative capacity in male smokers and non-smokers. British Journal of Nutrition 97:349-356. doi Doi 10.1017/S0007114507328626.

36. Roller M, Clune Y, Collins K, Rechkemmer G, Watzl B (2007) Consumption of prebiotic inulin enriched with oligofructose in combination with the probiotics Lactobacillus rhamnosus and Bifidobacterium lactis has minor effects on selected immune parameters in polypectomised and colon cancer patients. British Journal of Nutrition 97:676-684. doi Doi $10.1017 /$ S0007114507450292.

37. Lefevre $M$, Jonnalagadda S (2012) Effect of whole grains on markers of subclinical inflammation. Nutr Rev 70:387-396. doi Doi 10.1111/J.1753-4887.2012.00487.X.

38. Langkamp-Henken B, Nieves C, Culpepper T, Radford A, Girard SA, Hughes C, Christman MC, Mai V, Dahl WJ, Boileau T, Jonnalagadda SS, Thielecke F (2012) Fecal Lactic Acid Bacteria Increased in Adolescents Randomized to Whole-Grain but Not Refined-Grain Foods, whereas Inflammatory Cytokine Production Decreased Equally with Both Interventions. Journal of Nutrition 142:20252032. doi Doi 10.3945/Jn.112.164996.

39. Fukagawa NK, Anderson JW, Hageman G, Young VR, Minaker KL (1990) High-Carbohydrate, HighFiber Diets Increase Peripheral Insulin Sensitivity in Healthy-Young and Old Adults. American Journal of Clinical Nutrition 52:524-528.

40. Tighe P, Duthie G, Vaughan N, Brittenden J, Simpson WG, Duthie S, Mutch W, Wahle K, Horgan G, Thies $F(2010)$ Effect of increased consumption of whole-grain foods on blood pressure and other cardiovascular risk markers in healthy middle-aged persons: a randomized controlled trial. American Journal of Clinical Nutrition 92:733-740. doi Doi 10.3945/Ajcn.2010.29417.

41. Atkinson FS, Foster-Powell K, Brand-Miller JC (2008) International Tables of Glycemic Index and Glycemic Load Values: 2008. Diabetes Care 31:2281-2283. doi Doi 10.2337/Dc08-1239.

42. Juanola-Falgarona M, Salas-Salvado J, Ibarrola-Jurado N, Rabassa-Soler A, Diaz-Lopez A, GuaschFerre M, Hernandez-Alonso P, Balanza R, Bullo M (2014) Effect of the glycemic index of the diet on weight loss, modulation of satiety, inflammation, and other metabolic risk factors: a randomized controlled trial. American Journal of Clinical Nutrition 100:27-35. doi Doi 10.3945/Ajcn.113.081216.

43. Koh-Banerjee P, Rimm EB (2003) Whole grain consumption and weight gain: a review of the epidemiological evidence, potential mechanisms and opportunities for future research. Proceedings of the Nutrition Society 62:25-29. doi Doi 10.1079/Pns2002232.

44. Esposito K, Nappo F, Giugliano F, Di Palo C, Ciotola M, Barbieri M, Paolisso G, Giugliano D (2003) Meal modulation of circulating interleukin 18 and adiponectin concentrations in healthy subjects and in patients with type 2 diabetes mellitus. American Journal of Clinical Nutrition 78:1135-1140.

45. Anson NM, Aura AM, Selinheimo E, Mattila I, Poutanen K, van den Berg R, Havenaar R, Bast A, Haenen GRMM (2011) Bioprocessing of Wheat Bran in Whole Wheat Bread Increases the Bioavailability of Phenolic Acids in Men and Exerts Antiinflammatory Effects ex Vivo. Journal of Nutrition 141:137-143. doi Doi 10.3945/Jn.110.127720.

46. Hallfrisch J, Behall KM (2000) Mechanisms of the effects of grains on insulin and glucose responses. Journal of the American College of Nutrition 19:320S-325S. 
574 FIGURE 1. Intervention study design of a randomized controlled crossover study in which 33 575 subjects received a whole-grain (WG) diet $(>80 \mathrm{~g} / \mathrm{d}$ of $\mathrm{WG})$ and a refined grain diet $(<16 \mathrm{~g} / \mathrm{d}$ of 576 WG) for a period of 6 weeks each. Blood and saliva samples were collected from each volunteer 577 before and after each intervention arm.

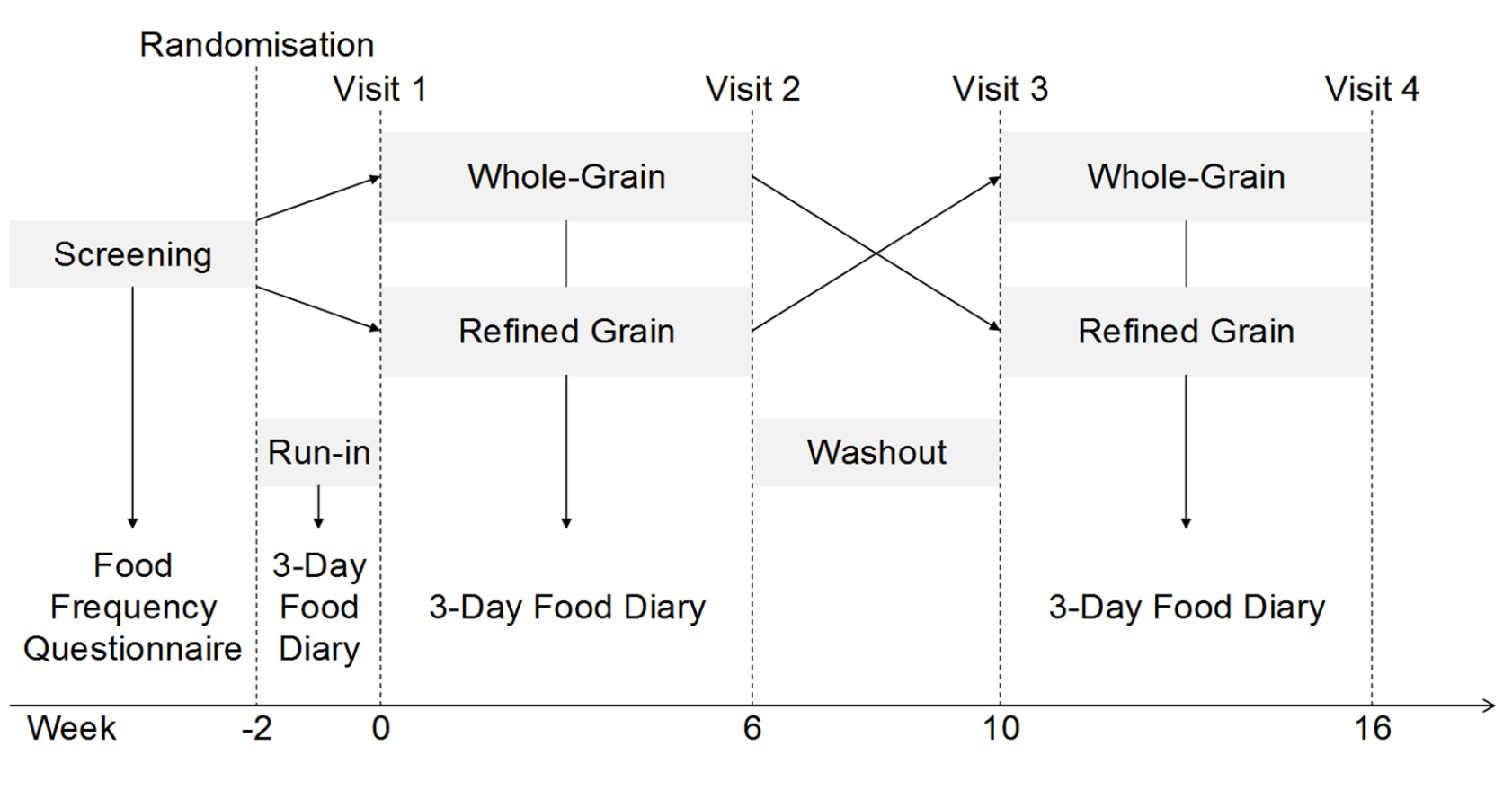


Table 1 Baseline characteristics of volunteers and baseline measurements of plasma lipids and glucose, body composition, blood pressure (BP) and alcohol consumption ${ }^{1}$

\begin{tabular}{lccc}
\hline Parameter $($ unit $)$ & Total volunteers & Male volunteers & Female volunteers \\
\hline $\mathrm{n}$ & 33 & 12 & 21 \\
Age $($ years $)$ & $48.8 \pm 1.1$ & $51.8 \pm 2.2$ & $47.1 \pm 1.1$ \\
Cholesterol $(\mathrm{mmol} / \mathrm{L})$ & $5.2 \pm 0.2$ & $5.5 \pm 0.3$ & $5.1 \pm 0.2$ \\
LDL cholesterol $(\mathrm{mmol} / \mathrm{L})$ & $3.6 \pm 0.2$ & $3.9 \pm 0.3$ & $3.5 \pm 0.2$ \\
HDL cholesterol $(\mathrm{mmol} / \mathrm{L})$ & $1.4 \pm 0.1$ & $1.3 \pm 0.1$ & $1.4 \pm 0.1$ \\
Triglycerides $(\mathrm{mmol} / \mathrm{L})$ & $1.1 \pm 0.1$ & $1.4 \pm 0.2$ & $1.0 \pm 0.1$ \\
Glucose $(\mathrm{mmol} / \mathrm{L})$ & $5.4 \pm 0.1$ & $5.6 \pm 0.2$ & $5.2 \pm 0.1$ \\
Weight $(\mathrm{kg})$ & $77.3 \pm 2.5$ & $87.6 \pm 4.3$ & $71.4 \pm 2.2$ \\
BMI $\left(\mathrm{kg} / \mathrm{m}^{2}\right)$ & $27.9 \pm 0.7$ & $28.9 \pm 1.5$ & $27.4 \pm 0.7$ \\
\% Body fat & $32.0 \pm 1.5$ & $25.4 \pm 2.3$ & $35.9 \pm 1.4$ \\
Waist circumference $(\mathrm{cm})$ & $97.3 \pm 1.9$ & $102.7 \pm 3.6$ & $94.2 \pm 2.0$ \\
Systolic BP $(\mathrm{mmHg})$ & $128.7 \pm 2.1$ & $135.4 \pm 3.3$ & $124.8 \pm 2.3$ \\
Diastolic BP $(\mathrm{mmHg})$ & $81.7 \pm 1.4$ & $82.9 \pm 2.5$ & $81.0 \pm 1.7$ \\
Alcohol consumption $($ units/week $)$ & $2.9 \pm 0.6$ & $4.8 \pm 1.2$ & $1.8 \pm 0.6$ \\
\hline
\end{tabular}

${ }^{1}$ Values are means \pm SEM. Six volunteers suffered from mild hay fever and occasionally took antihistamines or used corticosteroid nasal sprays and four volunteers occasionally consumed prebiotic/probiotic supplements. During the study, including the 2-week run-in period, however, no volunteers reported taking medication for hay fever or consuming prebiotic/probiotic supplements. 
Table 2 Energy, macronutrient, total fibre and WG intake and plasma ARs before the start of the study (habitual) and at the end of the RG and WG interventions $(\mathrm{n}=33)$

\begin{tabular}{|c|c|c|c|c|c|c|c|c|c|}
\hline \multirow[b]{3}{*}{ Parameter } & \multicolumn{9}{|c|}{ Energy $(\mathrm{kJ} / / \mathrm{d})$, macronutrient $(\mathrm{g} / \mathrm{d})$, fibre $(\mathrm{AOAC}, \mathrm{g} / \mathrm{d})$ and WG $(\mathrm{g} / \mathrm{d})$ intake and plasma ARs $(\mathrm{nM})$} \\
\hline & \multicolumn{2}{|c|}{ Habitual } & \multicolumn{2}{|c|}{ RG intervention } & \multicolumn{2}{|c|}{ WG intervention } & \multicolumn{3}{|c|}{$P$-values (Tukey Simultaneus Tests) } \\
\hline & Mean & (SD) & Mean & (SD) & Mean & (SD) & Habitual vs RG & Habitual vs WG & RG vs WG \\
\hline Energy & 8,765 & $(2,536)$ & 8,686 & $(1,937)$ & 8,556 & $(2,088)$ & 0.989 & 0.924 & 0.969 \\
\hline Protein & 86.0 & (27.9) & 81.1 & (21.9) & 82.9 & $(17.5)$ & 0.677 & 0.853 & 0.950 \\
\hline Fat & 83.9 & (35.7) & 80.2 & $(25.1)$ & 72.9 & (27.9) & 0.874 & 0.310 & 0.594 \\
\hline Saturated fat & 30.4 & (14.0) & 32.0 & (12.6) & 27.7 & $(13.0)$ & 0.878 & 0.692 & 0.396 \\
\hline Carbohydrate & 234.3 & (69.9) & 240.8 & $(56.7)$ & 223.6 & (60.6) & 0.911 & 0.773 & 0.518 \\
\hline Sugars & 103.1 & (48.3) & 96.4 & (32.4) & 95.9 & $(46.2)$ & 0.803 & 0.778 & 0.999 \\
\hline Fibre (AOAC) & 17.9 & (7.1) & 15.5 & (6.0) & 25.8 & (7.6) & 0.364 & $<0.001$ & $<0.001$ \\
\hline WG & 27.7 & (25.6) & 0.1 & (0.6) & 168.4 & $(63.3)$ & 0.017 & $<0.001$ & $<0.001$ \\
\hline Plasma ARs & 59.8 & (66.6) & 38.1 & $(29.4)$ & 161.1 & (176.8) & 0,712 & 0.001 & $<0.001$ \\
\hline
\end{tabular}

WG, whole-grain; ARs, alkylresorcinols; RG, refined grain

$P$-values $<0.05$ are highlighted in bold 
Table 3 Effects of WG and RG interventions on naïve and memory cells subpopulations of $\mathrm{CD}^{+}{ }^{+}$and $\mathrm{CD} 8^{+} \mathrm{T}$ lymphocytes $(\mathrm{n}=33)$

\begin{tabular}{|c|c|c|c|c|c|c|c|c|c|c|c|c|c|c|c|}
\hline \multirow[b]{4}{*}{ Parameter } & \multicolumn{15}{|c|}{ Nä̈ve and memory cells $\%$ of parent $T$ lymphocyte subset } \\
\hline & \multicolumn{5}{|c|}{ RG intervention } & \multicolumn{5}{|c|}{ WG intervention } & \multicolumn{5}{|c|}{$\Delta$ intervention } \\
\hline & \multicolumn{2}{|c|}{ Baseline } & \multicolumn{2}{|c|}{ End } & \multirow{2}{*}{$\frac{\text { Comparison }}{P \text {-value }}$} & \multicolumn{2}{|c|}{ Baseline } & \multicolumn{2}{|c|}{ End } & \multirow{2}{*}{$\begin{array}{c}\text { Comparison } \\
P \text {-value }\end{array}$} & \multicolumn{2}{|c|}{$\Delta \mathbf{R G}$} & \multicolumn{2}{|c|}{$\Delta W G$} & \multirow{2}{*}{$\frac{\text { Comparison }}{P \text {-value }}$} \\
\hline & Mean & $(\mathbf{S E M})$ & Mean & (SEM) & & Mean & (SEM) & Mean & (SEM) & & Mean & (SEM) & Mean & (SEM) & \\
\hline \multicolumn{16}{|l|}{$\mathrm{CD4}^{+}$} \\
\hline Naïve & 30.2 & $(2.1)$ & 30.3 & $(2.0)$ & 1.000 & 29.4 & $(2.1)$ & 30.0 & $(2.0)$ & 0.998 & 0.1 & $(0.8)$ & 0.5 & $(0.7)$ & 0.787 \\
\hline $\mathrm{Tcm}$ & 25.5 & (1.5) & 24.6 & (1.3) & 0.879 & 24.4 & (1.3) & 24.9 & (1.5) & 0.968 & -0.8 & (0.6) & 0.5 & $(0.5)$ & 0.062 \\
\hline Tem & 39.2 & (2.0) & 39.9 & (2.0) & 0.976 & 40.7 & (2.0) & 40.0 & (2.0) & 0.992 & 0.6 & (1.0) & -0.7 & (0.9) & 0.345 \\
\hline Temra & 5.1 & $(0.7)$ & 5.2 & $(0.7)$ & 0.996 & 5.5 & $(0.7)$ & 5.2 & $(0.6)$ & 0.948 & 0.1 & (0.4) & -0.3 & $(0.3)$ & 0.328 \\
\hline \multicolumn{16}{|l|}{$\mathrm{CD8}^{+}$} \\
\hline Naïve & 23.0 & $(2.2)$ & 21.7 & (2.1) & 0.968 & 22.0 & (2.1) & 22.4 & $(2.2)$ & 0.998 & -1.3 & $(0.9)$ & 0.4 & $(0.8)$ & 0.173 \\
\hline $\mathrm{Tcm}$ & 4.7 & $(0.5)$ & 4.6 & (0.4) & 1.000 & 4.7 & (0.6) & 4.8 & $(0.5)$ & 0.999 & 0.0 & $(0.2)$ & 0.1 & $(0.3)$ & 0.783 \\
\hline Tem & 56.7 & (2.3) & 58.0 & $(2.3)$ & 0.975 & 57.8 & $(2.2)$ & 57.2 & (2.3) & 0.996 & 1.3 & $(0.8)$ & -0.6 & $(0.8)$ & 0.143 \\
\hline Temra & 15.6 & (1.7) & 15.7 & (1.7) & 1.000 & 15.5 & (1.7) & 15.6 & (1.6) & 1.000 & 0.0 & $(0.5)$ & 0.1 & $(0.5)$ & 0.896 \\
\hline
\end{tabular}

WG, whole-grain; RG, refined grain; Tcm, central memory T lymphocytes; Tem, effector memory T lymphocytes; Temra, RA ${ }^{+}$effector memory T lymphocytes

Within the RG and WG interventions, comparisons were performed between baseline and end measurements, while within $\Delta$ intervention $\Delta \mathrm{RG}$ was compared against $\Delta \mathrm{WG}$. $P$-values $<0.1$ are highlighted in bold 
Table 4 Effects of WG and RG interventions on activation (expression of CD69) of $\mathrm{CD}^{+}$and $\mathrm{CD} 8^{+} \mathrm{T}$ lymphocyte subsets following ex vivo stimulation $(\mathrm{n}=33)$

\begin{tabular}{|c|c|c|c|c|c|c|c|c|c|c|c|c|c|c|c|}
\hline \multirow[b]{4}{*}{ Parameter } & \multicolumn{15}{|c|}{ CD69 $^{+}$cells \% of parent T lymphocyte subset } \\
\hline & \multicolumn{5}{|c|}{ RG intervention } & \multicolumn{5}{|c|}{ WG intervention } & \multicolumn{5}{|c|}{$\Delta$ intervention } \\
\hline & \multicolumn{2}{|c|}{ Baseline } & \multicolumn{2}{|c|}{ End } & \multirow{2}{*}{$\frac{\text { Comparison }}{P \text {-value }}$} & \multicolumn{2}{|c|}{ Baseline } & \multicolumn{2}{|c|}{ End } & \multirow{2}{*}{$\begin{array}{c}\text { Comparison } \\
P \text {-value }\end{array}$} & \multicolumn{2}{|c|}{$\Delta \mathbf{R G}$} & \multicolumn{2}{|c|}{$\Delta W G$} & \multirow{2}{*}{$\begin{array}{c}\text { Comparison } \\
P \text {-value }\end{array}$} \\
\hline & Mean & (SEM) & Mean & (SEM) & & Mean & (SEM) & Mean & (SEM) & & Mean & (SEM) & Mean & (SEM) & \\
\hline \multicolumn{16}{|l|}{$\mathrm{CD4}^{+}$} \\
\hline Unstimulated & 1.6 & $(0.2)$ & 1.3 & $(0.2)$ & 0.932 & 1.9 & $(0.4)$ & 1.3 & $(0.1)$ & 0.255 & -0.2 & $(0.3)$ & -0.6 & $(0.4)$ & 0.293 \\
\hline ConA & 6.9 & $(0.9)$ & 8.0 & $(1.1)$ & 0.654 & 8.6 & (1.3) & 7.0 & $(0.9)$ & 0.72 & 1.3 & $(1.0)$ & -1.6 & $(1.2)$ & 0.079 \\
\hline CytoStim & 54.1 & (2.6) & 56.3 & $(2.5)$ & 0.783 & 53.9 & (2.6) & 53.4 & $(2.2)$ & 1.000 & 1.8 & $(1.2)$ & -0.4 & (1.6) & 0.535 \\
\hline \multicolumn{16}{|l|}{$\mathrm{CD8}^{+}$} \\
\hline Unstimulated & 2.4 & $(0.2)$ & 2.4 & $(0.2)$ & 1.000 & 2.5 & $(0.2)$ & 2.6 & $(0.3)$ & 0.99 & 0.1 & $(0.2)$ & 0.1 & $(0.3)$ & 0.787 \\
\hline ConA & 11.3 & (1.3) & 12.4 & (1.1) & 0.883 & 11.8 & (1.5) & 11.5 & $(1.2)$ & 0.999 & 1.3 & $(0.8)$ & -0.3 & $(0.8)$ & 0.174 \\
\hline CytoStim & 55.1 & (2.2) & 56.1 & (2.2) & 0.992 & 54.2 & (2.1) & 53.9 & (1.9) & 1.000 & 0.5 & (1.1) & -0.3 & (1.5) & 0.855 \\
\hline
\end{tabular}

WG, whole-grain; RG, refined grain; ConA, Concanavalin A; CytoStim, Miltenyi Biotec Ltd.-130-092-173

Within the RG and WG interventions, comparisons were performed between baseline and end measurements, while within $\Delta$ intervention $\Delta \mathrm{RG}$ was compared against $\Delta \mathrm{WG} . P$-values $<0.1$ are highlighted in bold 
Table 5 Effects of WG and RG interventions on serum concentrations of cytokines and CRP $(n=33)$

Concentration in serum (ng/l)

\section{RG intervention}

WG intervention

$\Delta$ intervention

\begin{tabular}{|c|c|c|c|c|c|c|c|c|c|c|c|c|c|c|c|}
\hline \multirow[b]{2}{*}{ Parameter } & \multicolumn{2}{|c|}{ Baseline } & \multicolumn{2}{|c|}{ End } & \multirow{2}{*}{$\begin{array}{c}\text { Comparison } \\
P \text {-value }\end{array}$} & \multicolumn{2}{|c|}{ Baseline } & \multicolumn{2}{|c|}{ End } & \multirow{2}{*}{$\begin{array}{c}\text { Comparison } \\
P \text {-value }\end{array}$} & \multicolumn{2}{|c|}{$\Delta R G$} & \multicolumn{2}{|c|}{$\Delta W G$} & \multirow{2}{*}{$\begin{array}{c}\text { Comparisor } \\
P \text {-value }\end{array}$} \\
\hline & Mean & (SEM) & Mean & (SEM) & & Mean & (SEM) & Mean & (SEM) & & Mean & (SEM) & Mean & (SEM) & \\
\hline IL-10 & 0.8 & $(0.1)$ & 0.9 & $(0.1)$ & 0.996 & 1.1 & $(0.3)$ & 0.8 & $(0.1)$ & 0.326 & 0.1 & $(0.1)$ & -0.3 & $(0.2)$ & 0.075 \\
\hline IL- $1 \beta$ & 1.3 & $(0.1)$ & 1.3 & $(0.1)$ & 0.905 & 1.3 & $(0.1)$ & 1.3 & $(0.1)$ & 0.94 & 0.1 & $(0.1)$ & 0.1 & $(0.0)$ & 0.888 \\
\hline IL-6 & 1.3 & $(0.2)$ & 1.4 & $(0.2)$ & 0.99 & 1.2 & $(0.2)$ & 1.2 & $(0.1)$ & 0.999 & 0.1 & $(0.2)$ & -0.1 & $(0.1)$ & 0.702 \\
\hline IL-8 & 9.9 & $(0.6)$ & 10.2 & $(0.8)$ & 0.931 & 10.1 & $(0.8)$ & 10.4 & $(0.8)$ & 0.996 & 0.3 & $(0.5)$ & 0.3 & $(0.5)$ & 0.649 \\
\hline TNF- $\alpha$ & 10.5 & $(0.5)$ & 10.7 & $(0.5)$ & 0.937 & 10.8 & (0.4) & 10.8 & (0.6) & 0.995 & 0.2 & $(0.3)$ & 0.0 & (0.4) & 0.381 \\
\hline $\operatorname{CRP}\left(\times 10^{3}\right)$ & 1.7 & $(0.3)$ & 1.8 & $(0.3)$ & 0.948 & 2.2 & $(0.5)$ & 1.6 & $(0.4)$ & 0.248 & 0.1 & $(0.2)$ & -0.6 & $(0.4)$ & 0.099 \\
\hline
\end{tabular}

WG, whole-grain; RG, refined grain; CRP, C-reactive protein; TNF, tumor necrosis factor

Within the RG and WG interventions, comparisons were performed between baseline and end measurements, while within $\Delta$ intervention $\Delta \mathrm{RG}$ was compared against $\Delta$ WG. $P$-values $<0.1$ are highlighted in bold 
Table 6 Effects of WG and RG interventions on serum concentration of glucose metabolism related proteins $(\mathrm{n}=33)$

\begin{tabular}{|c|c|c|c|c|c|c|c|c|c|c|c|c|c|c|c|}
\hline \multirow[b]{4}{*}{ Parameter } & \multicolumn{15}{|c|}{ Concentration in serum (ng/l) } \\
\hline & \multicolumn{5}{|c|}{ RG intervention } & \multicolumn{5}{|c|}{ WG intervention } & \multicolumn{5}{|c|}{$\Delta$ intervention } \\
\hline & \multicolumn{2}{|c|}{ Baseline } & \multicolumn{2}{|c|}{ End } & \multirow{2}{*}{$\frac{\text { Comparison }}{P \text {-value }}$} & \multicolumn{2}{|c|}{ Baseline } & \multicolumn{2}{|c|}{ End } & \multirow{2}{*}{$\frac{\text { Comparison }}{P \text {-value }}$} & \multicolumn{2}{|c|}{$\Delta \mathbf{R G}$} & \multicolumn{2}{|c|}{$\Delta W G$} & \multirow{2}{*}{$\frac{\text { Comparison }}{P \text {-value }}$} \\
\hline & Mean & (SEM) & Mean & (SEM) & & Mean & (SEM) & Mean & (SEM) & & Mean & (SEM) & Mean & (SEM) & \\
\hline C-Peptide & 889.8 & $(63.9)$ & 982.8 & $(83.6)$ & 0.535 & 940.9 & $(75.5)$ & 878.7 & $(70.3)$ & 0.846 & 93.0 & $(69.0)$ & -62.3 & $(61.6)$ & 0.099 \\
\hline Ghrelin & 918.0 & $(55.4)$ & 916.0 & $(58.0)$ & 0.999 & 880.7 & $(50.2)$ & 914.8 & $(54.0)$ & 0.868 & -1.9 & $(31.8)$ & 34.1 & $(20.9)$ & 0.193 \\
\hline GIP & 186.1 & $(23.5)$ & 193.4 & $(31.7)$ & 0.999 & 231.8 & $(68.8)$ & 183.5 & $(31.9)$ & 0.745 & 7.3 & $(20.5)$ & -48.4 & $(38.8)$ & 0.239 \\
\hline GLP-1 & 347.6 & $(36.2)$ & 348.3 & $(36.5)$ & 1.000 & 351.6 & $(36.7)$ & 348.1 & $(36.5)$ & 1.000 & 0.8 & (4.4) & -3.5 & (5.9) & 0.516 \\
\hline Glucagon & 552.0 & $(12.4)$ & 552.5 & $(12.3)$ & 1.000 & 564.3 & (12.8) & 555.2 & $(13.3)$ & 0.903 & 0.5 & $(4.9)$ & -9.1 & $(4.1)$ & 0.151 \\
\hline Insulin & 438.2 & $(45.4)$ & 491.1 & $(53.7)$ & 0.583 & 491.6 & $(58.6)$ & 434.8 & $(41.9)$ & 0.614 & 52.9 & $(43.5)$ & -56.8 & $(50.6)$ & 0.075 \\
\hline Leptin $\left(\times 10^{3}\right)$ & 12.1 & (1.6) & 11.6 & (1.4) & 0.932 & 12.3 & (1.4) & 11.0 & (1.3) & 0.589 & -0.4 & $(0.5)$ & -1.3 & $(0.6)$ & 0.489 \\
\hline PAI-1 $\left(\mathrm{x} 10^{3}\right)$ & 41.7 & (2.0) & 41.9 & $(1.5)$ & 1.000 & 47.3 & (3.1) & 41.9 & (1.3) & 0.074 & 0.2 & (1.9) & -5.4 & (3.1) & 0.068 \\
\hline Resistin (x 10 $0^{3}$ ) & 4.0 & $(0.3)$ & 4.2 & $(0.3)$ & 0.904 & 4.1 & $(0.3)$ & 4.1 & $(0.3)$ & 1.000 & 0.2 & $(0.1)$ & -0.1 & $(0.1)$ & 0.136 \\
\hline Visfatin (x 10 $\left.0^{3}\right)$ & 4.4 & $(0.9)$ & 4.6 & (1.0) & 1.000 & 6.8 & (3.1) & 4.8 & $(1.2)$ & 0.74 & 0.2 & $(0.2)$ & -2.0 & (2.0) & 0.28 \\
\hline Adiponectin $\left(\mathrm{x} 10^{6}\right)$ & 2.8 & $(0.3)$ & 2.7 & $(0.3)$ & 0.99 & 2.8 & $(0.3)$ & 2.6 & $(0.3)$ & 0.834 & -0.1 & $(0.2)$ & -0.2 & $(0.2)$ & 0.538 \\
\hline $\operatorname{Adipsin}\left(\mathrm{x} 10^{3}\right)$ & 660.0 & (31.4) & 615.5 & $(36.3)$ & 0.395 & 643.2 & $(35.3)$ & 656.7 & $(36.6)$ & 0.973 & -44.5 & (29.5) & 13.6 & $(25.3)$ & 0.071 \\
\hline
\end{tabular}

WG, whole-grain; RG, refined grain; GIP, gastric inhibitory polypeptide; GLP-1, glucagon-like peptide-1; PAI-1, plasminogen activator inhibitor-1

Within the RG and WG interventions, comparisons were performed between baseline and end measurements, while within $\Delta$ intervention $\Delta$ RG was compared against $\Delta$ WG. $P$-values $<0.1$ are highlighted in bold 
